\title{
A CUSP CLOSING THEOREM
}

\author{
VIKTOR SCHROEDER
}

(Communicated by Jonathan M. Rosenberg)

\begin{abstract}
Using a modification of a cusp closing result of Thurston, we construct compact Riemannian manifolds of nonpositive sectional curvature which have rank one (in the sense of Brin, Ballmann, and Eberlein) but which contain embedded flat tori of codimension 2 . The metric can even be made analytic.
\end{abstract}

\section{INTRODUCTION}

Using a modification of a cusp closing result of Thurston [T, G], we construct compact Riemannian manifolds of nonpositive sectional curvature which have rank 1 (in the sense of [BBE]) but contain flat tori of codimension 2. There are also analytic metrics of this type. In particular we obtain the following result:

Theorem. For every integer $n \geq 4$ there exists a compact real analytic $n$ dimensional Riemannian manifold $M$ with sectional curvature $K \leq 0$ which contains a totally geodesic embedded flat torus $T \subset M$ of codimension 2 such that the sectional curvature is strictly negative on all 2-planes which are not tangent to $T$.

If $M$ is a manifold of this type, then a geodesic which is not contained in $T$, does not allow nontrivial parallel Jacobi vector fields and thus the rank of $M$ is 1 in the sense of [BBE].

Heintze has constructed $C^{\infty}$-infinity manifolds which contain a codimension 1 flat torus $T$ such that the curvature is negative for all planes not tangent to $T$. Using methods from sheaf theory (as in [BG]) one can modify the metric to be analytic with the same properties (comp. [S]).

The idea of our construction is a modification of a cusp closing Theorem of Thurston. His construction arises in the context of hyperbolic 3-manifolds. Let $W$ be a complete 3-dimensional hyperbolic $(K \equiv-1)$ manifold with a cusp. The cusp $C$ has topologically the structure $C=T^{2} \times[0, \infty)$. Now cut off the cusp to obtain a manifold $W_{c}$ with a boundary torus $T^{2}$. Onto this boundary we glue a tube around a closed geodesic. The tube is topologically $D \times S^{1}$ where

Received by the editors September 2, 1988.

1980 Mathematics Subject Classification (1985 Revision). Primary 53C20, 58F17.

Key words and phrases. Nonpositive curvature, rank, analytic Riemannian metric. 
$D$ is a disc. Thus $\partial$ (tube) $=S^{1} \times S^{1}=T^{2}$ and one can identify $\partial$ (tube) with $\partial\left(W_{c}\right)$ by a diffeomorphism of $T^{2}$.

We now examine this situation from a metric viewpoint. The cusp has metrically the structure of a warped product $C=[0, \infty) \times{ }_{g} \mathbf{R}^{2} / \Gamma$ with the warping function $g:[0, \infty) \rightarrow \mathbf{R}, g(t)=e^{-t}$, i.e. the metric is given by $d t^{2}+g^{2}(t) d s^{2}$ where $d s^{2}$ is the standard Euclidean metric on $\mathbf{R}^{2}$. The group $\Gamma$ is isomorphic to $\mathbf{Z}^{2}$ and operates as a lattice on $\mathbf{R}^{2}$. The boundary of the cusp is a quotient of a horosphere.

Next we give a metric model for the tube. For this purpose, consider the distance tube $T_{r}(c)$ of a geodesic $c$ in hyperbolic 3-space $H^{3}$. Then the tube around the geodesic is $T_{r}(c) / \mathbf{Z}$, where $\mathbf{Z}$ is generated by an isometry $\alpha$ of $H^{3}$ which translates the geodesic $c$. For $r \rightarrow \infty$, the boundary $\partial T_{r}(c)$ converges metrically to a horosphere. Let $r$ be very large. Then it is not difficult to construct an operation of $\mathbf{Z}$ on $T_{r}(c)$ such that $\partial\left(T_{r}(c) / \mathbf{Z}\right)$ is metrically close to $\partial C$. Thus one can glue the tube onto $\partial\left(M_{c}\right)$ with a "small" metric singularity. After smoothing we obtain a metric with curvature close to -1 .

Using different methods Thurston can actually show the existence of constant curvature metrics on these manifolds. We start more generally with an $n$ dimensional hyperbolic manifold of finite volume and a cusp diffeomorphic to $C=T^{n-1} \times[0, \infty)$. We show that one can close the cusp by a tube $D \times T^{n-2}$ around a flat torus of codimension 2. We do this construction in two steps. First, in $\S 2$, we construct suitable metrics on the universal covering of the tube, i.e., on $D \times \mathbf{R}^{n-2}$. In the second step, $\S 3$, we construct an operation of the group $\mathbf{Z}^{n-2}$ on $D \times R^{n-2}$ such that $\partial\left(D \times \mathbf{R}^{n-2} / \mathbf{Z}^{n-2}\right)$ is metrically near to $\partial C$. Then the glueing and smoothing of the metric is possible.

\section{Metrics on A TUBe}

Consider the orthogonal decomposition of $\mathbf{R}^{n}$ as $\mathbf{R}^{2} \times \mathbf{R}^{n-2}$ and on $\mathbf{R}^{2}$ polar coordinates $[0, \infty) \times S^{1}$. Thus we have cylindrical coordinates $[0, \infty) \times S^{1} \times$ $\mathbf{R}^{n-2}$ on $\mathbf{R}^{n}$. We study metrics of the type $d t^{2}+h^{2}(t) d \varphi^{2}+g^{2}(t) d s^{2}$ where $d t^{2}, d \varphi^{2}$ and $d s^{2}$ are the standard metrics on $[0, \infty), S^{1}$ and $\mathbf{R}^{n-2}$. One can describe this metric also as a doubled warped product $X=\left([0, \infty) \times{ }_{g} \mathbf{R}^{n-2}\right) \times_{f}$ $S^{1}$, where $g$ is a warped function on $[0, \infty)$ and $f$ is a warping function on $[0, \infty) \times \mathbf{R}^{n-2}$ depending only on the first coordinate, i.e.,. $f(t, p)=h(t)$.

In order to obtain a smooth metric on $\mathbf{R}^{n}$ we have to assume

(1) $g$ is the restriction of a smooth positive even function on $\mathbf{R}$ and $h$ is the restriction of a smooth odd function with derivative 1 at the origin. To obtain metrics of nonpositive sectional curvature we assume $(2) g_{\mid[0, \infty)}$ and $h_{\mid[0, \infty)}$ are convex functions. We first show that the conditions (1) and (2) really imply that the curvature of $X$ is nonpositive.

Since $g$ is an even convex function, we have $g^{\prime}(0)=0, g^{\prime}(t) \geq 0$ and $g^{\prime \prime}(t) \geq 0$ for $t \in[0, \infty)$. Thus the warped product formula [BO, 7.5] implies 
that the curvature of $[0, \infty) \times{ }_{g} \mathbf{R}^{n-2}$ is nonpositive. Furthermore the curvature is negative in points $(t, p)$ with $g^{\prime}(t)>0$. If $g^{\prime}\left(t_{0}\right)=0$, then $\left\{t_{0}\right\} \times \mathbf{R}^{n-2}$ is a totally geodesic submanifold. Since $g^{\prime}(0)=0$ we see that $[0, \infty) \times{ }_{g} \mathbf{R}^{n-2}$ has totally geodesic boundary. Note that the projection $p_{1}(t, p)=t$ is equal to the distance function from the boundary. Since the boundary is totally geodesic, this function is convex (see e.g. [ BGS] $\S 1$ ).

To prove that the curvature of $X$ is nonpositive we have to show that $f$ is convex on $Y=[0, \infty) \times{ }_{g} \mathbf{R}^{n-2}$. Let therefore $c:[0,1] \rightarrow Y$ be a geodesic. Then $f \circ c(s)=h\left(p_{1} \circ c(s)\right)$. We know already that $\alpha(s)=p_{1} \circ c(s)$ is convex. Since $h$ is convex and $h^{\prime}(0)=1$ we have $h^{\prime}(t)>1$ for all $t>0$. Thus $(f \circ c)^{\prime \prime}(s)=h^{\prime \prime}(\alpha(s)) \cdot \alpha^{\prime}(s)^{2}+h^{\prime}(\alpha(s)) \cdot \alpha^{\prime \prime}(s) \geq 0$.

This discussion shows that $X$ has nonpositive curvature and contains a flat totally geodesic subspace $\{0\} \times \mathbf{R}^{n-2}$. The function $t$ describes the distance to this flat. If the functions $g$ and $h$ are strictly convex on $(0, \infty)$, then the curvature of $X$ is strictly negative on all 2-planes which are not tangent to the flat $\{0\} \times \mathbf{R}^{n-2}$.

For $r>0$ let $T_{r} \subset X$ be the $r$-distance tube of the flat, i.e., $T_{r}=([0, r] \times g$ $\left.\mathbf{R}^{n-2}\right) \times{ }_{f} S^{1}$. Clearly the isometry group of $T_{r}$ is isomorphic to $\operatorname{Iso}\left(S^{1}\right) \times$ Iso $\left(\mathbf{R}^{n-2}\right)$ in a canonical way.

To adapt our tube to the constant curvature situation we require in addition

$$
g(t)=h(t)=e^{t} \text { for all } t \geq r_{0} \text { where } r_{0} \text { is some positive constant } .
$$

It is easy to construct functions which satisfy the conditions (1)-(3). For $r \geq$ $r_{0}$ consider $X_{r}:=X \backslash T_{r}$ and the universal covering space $\widetilde{X}_{r}$. Then $\tilde{X}_{r}=$ $(r, \infty) \times{ }_{f_{0}} \mathbf{R}^{n-1}$, where $f_{0}(t)=e^{t}$. Thus $\tilde{X}_{r}$ is a subset of the complete manifold $\mathbf{R} \times{ }_{f_{0}} \mathbf{R}^{n-1}$ which is nothing else than a warped product representation of the real hyperbolic space of constant curvature -1 . In this coordinate of the hyperbolic space the lines $\mathbf{R} \times\{p\}$ are asymptotic geodesics and the hypersurfaces $\left\{t_{0}\right\} \times$ $\mathbf{R}^{n-1}$ are the horospheres orthogonal to these geodesics. Thus $\tilde{X}_{r}$ is isometric to the complement of a horoball in hyperbolic space. This means that $\partial T_{r}$ is locally isometric to a horosphere HS in hyperbolic space. We will need this property in $\S 3$.

\section{CLOSING THE CUSP}

We consider now a complete hyperbolic $(K \equiv-1)$ manifold $W$ with a cusp diffeomorphic to $T^{n-1} \times[0, \infty)$. The cusp is isometric to $H B / \Gamma$, where $H B$ is a horoball in hyperbolic space and $\Gamma \simeq \mathbf{Z}^{n-1}$ is a lattice operating with compact quotient on the horosphere $H S=\partial H B$. One can write $H B=H S \times(0, \infty)$ with a suitable warped product metric. We cut off the cusp at the horosphere $H S \times\{1\} / \Gamma$ to obtain a manifold $W_{c}$ with boundary. The boundary has the collar $H S \times(0,1] / \Gamma$. We want to glue a tube $T_{r} / \Delta$ onto $M_{c}$, where $T_{r}$ is as 
in $\S 2$ and $\Delta$ is isomorphic to $\mathbf{Z}^{n-2}$ and operates as a lattice on the central flat $\mathbf{R}^{n-2}$ of $T_{r}$.

Note that by our construction for $r>r_{0}+1$, the boundary $\partial T_{r}$ has the 1-distance neighborhood $\partial T_{r} \times[0,1)$ which is locally isometric to $H S \times(0,1]$.

We will show below the following result:

For given $\varepsilon>0$ and $k \in \mathbf{N}$ there exists $r>r_{0}>0$ and an operations of $\Delta \simeq \mathbf{Z}^{n-2}$ on $T_{r}$ and a diffeomorphism $\varphi: \partial T_{r} / \Delta \rightarrow$

(*) $\quad H S / \Gamma$ such that $\varphi$ is $\varepsilon$-close to an isometry in the $C^{k}$-topology,

i.e. the metric on $\partial T_{r} / \Delta$ and pull back by $\varphi$ of the metric on $H S / \Gamma$ are $\varepsilon$-close.

Since $\partial T_{r} \times(0,1)$ is locally isometric to $H S \times(0,1)$ this implies for every $\varepsilon>0$ the existence of a diffeomorphism

$$
\psi: \partial T_{r} \times(0,1) / \Delta \rightarrow H S \times(0,1) / \Gamma
$$

of the form $\psi(p, t)=(\varphi(p), t)$ which is $\varepsilon$-close to an isometry in the $C^{k}$ topology, i.e., $\psi^{*} g_{1}$ is $\varepsilon$-close to $g_{0}$, where $g_{0}$ is the metric on $\partial T_{r} \times(0,1) / \Delta$ and $g_{1}$ the metric on $H S \times(0,1) / \Gamma$. Now we glue $M_{c}$ onto $T_{r} / \Delta$ by identifying $(p, t)$ with $(\varphi(p), t)$ on the overlapping set. To smooth the metric we take a suitable partition of unity on $(0,1)$ by functions $\alpha$ and $\beta$ and consider a metric of the type $\alpha g_{0}+\beta \psi^{*} g_{1}$ on the overlapping set. Since $g_{0}$ and $\psi^{*} g_{1}$ are $C^{k}$-close, also the new metric has curvature near to -1 (in particular negative curvature) on the overlapping set if we choose $\varepsilon$ and $r$ suitable. Therefore it is possible to close the cusp with a metric of curvature $K \leq 0$. In order to obtain an analytic metric on $M$ we choose the metric of $T_{r}$ analytic in some neighborhood of the flat $\{0\} \times \mathbf{R}^{n-2}$. For example let $g(t)=\cosh (t)$ and $h(t)=\sinh (t)$ for small $t \geq 0$. After glueing and smoothing we then obtain a $C^{\infty}$-metric $g_{\infty}$ on $M$ such that the curvature of $M$ is strictly negative for all planes which are not tangent to the codimension 2 torus $T$ and the metric is analytic in a neighborhood of $T$. We give $M$ the structure of an analytic manifold such that $T$ is an analytic submanifold. Using standard methods from sheaf theory (compare [BG] for details), one can approximate $g_{\infty}$ by an analytic metric $g_{\omega}$ in the $C^{\infty}$-topology, such that $g_{\omega}$ and $g_{\infty}$ coincide up to a fixed prescribed order $k$ on the submanifold $T$. We will indicate that this implies that the curvature of $g_{\omega}$ (for a sufficiently close approximation to $g_{\infty}$ ) is nonpositive and strictly negative for planes not tangent to $T$.

For this purpose, let $G$ be the grassmannbundle of 2-planes of $M$ equipped with the canonical Riemannian metric and let $H \subset G$ be the submanifold of planes tangent to $T$. We denote the curvature function of $g_{\infty}$ by $K_{\infty}: G \rightarrow$ $(-\infty, 0]$. Note that $H=\left\{K_{\infty}=0\right\}$ and $K_{\infty}$ is analytic in a neighborhood of $H$. Since $g_{\infty}$ and $g_{\omega}$ agree up to order $k$ on $T$, the function $K_{\infty}$ and the corresponding function $K_{\omega}$ agree up to order $k-2$ on $H$. Note that by analyticity in the neighborhood of $H$, not all derivatives normal to $H$ vanish 
for the function $K_{\infty}$. Indeed, if we choose $g$ and $h$ as above, the second derivative of $K_{\infty}$ is strictly negative in all directions normal to $H$. For $k \geq$ 4 and a sufficiently close approximation $g_{\omega}$ this implies that $K_{\omega}$ is strictly negative on $U \backslash H$, where $U$ is a neighborhood of $H$. Since $K_{\infty}<c<0$ on $G \backslash U$, also $K_{\omega}<0$ on $G \backslash U$ for a sufficiently close approximation.

It remains to prove $(*)$.

Note that $\partial T_{r}$ can be identified with $\mathbf{R}^{n-1} / 2 \pi e^{r} \mathbf{Z}$ where $2 \pi e^{r} \mathbf{Z}$ is the set $\left(2 \pi e^{r} k, 0, \ldots, 0\right) \in \mathbf{R}^{n-1}$ with $k \in \mathbf{Z}$. We first show that $(*)$ holds in the case $n=3$. Then $H S / \Gamma$ can be identified with a lattice $\mathbf{R}^{2} / \Gamma$, where $\Gamma$ is isomorphic to $\mathbf{Z}^{2}$ and a subgroup of $\mathbf{R}^{2}$. We choose generators $\alpha_{0}, \alpha_{1}$ of $\Gamma$ such that $\left|\alpha_{0}\right|$ is minimal in $\Gamma \backslash$ id and $\left|\alpha_{1}\right|$ is minimal in $\Gamma \backslash\left\langle\alpha_{1}\right\rangle$. We can choose coordinates such that $\alpha_{0}=(a, 0) \in \mathbf{R}^{2}$ and $\alpha_{1}=(b, c)$ with $a, b>0, c \neq 0$. Fix now some "large" integer $N$. Then choose $r$ such that $2 \pi e^{r}=N a-b$. Now we choose an isometry $\beta_{1}$ of $T_{r}, \beta_{1}$ of the form $\left(\beta_{1}^{\prime}, \beta_{1}^{\prime \prime}\right)$ in the decomposition $\operatorname{Iso}\left(T_{r}\right)=\operatorname{Iso}\left(S^{1}\right) \times \operatorname{Iso}(\mathbf{R})$, such that $\beta_{1}$ operates on $\partial T_{r}=\mathbf{R}^{2} / 2 \pi e^{r} \mathbf{Z}$ as the translation $(a, c / N)$. This determines $\beta$ uniquely. Since $c \neq 0$, we see that $\beta_{1}^{\prime \prime}$ is a translation of $\mathbf{R}$. Thus the group $\Delta=$ $\langle\beta\rangle$ is a group of isometries without fixed points on $T_{r}$. Note that $\beta_{1}^{N}$ is the translation by $(N a, c)$ which is equivalent to the translation $(b, c)$. Thus $\partial T_{r} / \Delta$ is isometric to $\mathbf{R}^{2} / \Gamma^{*}$ where $\Gamma^{*}$ is generated by $(a, c / N)$ and $(b, c)$. For $N$ sufficiently large, this operation is arbitrarily close to $\mathbf{R}^{2} / \Gamma$.

In the general case we may identify $H S / \Gamma$ with $\mathbf{R}^{n-1} / \Gamma, \Gamma$ a subgroup of $\mathbf{R}^{n-1}$ isomorphic to $\mathbf{Z}^{n-1}$. Choose generators $\alpha_{0}, \ldots, \alpha_{n-2}$ of $\Gamma$ such that $\left|\alpha_{0}\right|$ is minimal in $\Gamma \backslash$ id,$\left|\alpha_{1}\right|$ is minimal in $\Gamma \backslash\left\langle\alpha_{1}\right\rangle$, etc. For suitable coordinates $\alpha_{0}=(a, 0, \ldots, 0), \alpha_{1}=(b, c, 0, \ldots, 0)$. Choose as above $\beta_{1}$ as $\left(\beta_{1}^{\prime}, \beta_{1}^{\prime \prime}\right) \in \operatorname{Iso}\left(S^{1}\right) \times \operatorname{Iso}\left(\mathbf{R}^{n-2}\right)$ such that $\beta_{1}$ operates on $\partial T_{r}$ as translation $(a, c / N, 0, \ldots, 0)$. In addition choose $\beta_{j}=\left(\beta_{j}^{\prime}, \beta_{j}^{\prime \prime}\right), j=2, \ldots, n-2$ such that $\beta_{j}$ operates on $\partial T_{r}=\mathbf{R}^{n-1} / 2 \pi e^{r} \mathbf{Z}$ exactly as the translation $\alpha_{j}$. This determines $\beta_{j}$ uniquely. Since for $j \geq 1$ the translations $\alpha_{j}$ are independent from $\alpha_{0}=(a, 0, \ldots, 0)$, the isometries $\beta_{j}^{\prime \prime} \in \operatorname{Iso}\left(\mathbf{R}^{n-2}\right)$ are nontrivial translations. These translations are linearly independent since the $\alpha_{i}$ are independent. Thus $\Delta=\left\langle\beta_{1}, \ldots, \beta_{n-2}\right\rangle$ operates without fixed points on $T_{r}$ and the quotient $T_{t} / \Delta$ is a tube around a flat torus. Now $\partial T_{r} / \Delta$ is isometric to $\mathbf{R}^{n-2} / \Gamma^{*}$ where $\Gamma^{*}$ is generated by $\bar{\alpha}_{0}=(a, c / N, 0, \ldots, 0)$, $\alpha_{1}=(b, c, 0, \ldots, 0), \alpha_{2}, \ldots, \alpha_{n-2}$. For $N$ sufficiently large this lattice is arbitrarily close to $\mathbf{R}^{n-1} / \Gamma$. This proves (*).

\section{ACKNOWLEDGMENT}

This work was done while the author was professor visitante at IMPA in Rio de Janeiro. I would like to thank the institute for its hospitality and CNPq and GMD for financial support. 


\section{REFERENCES}

[BBE] W. Ballmann, M. Brin, and P. Eberlin, Structure of manifolds of nonpositive curvature. I, Ann. of Math. (2) 122 (1985), 171-203.

[BGS] W. Ballmann, M. Gromov and V. Schroeder, Manifolds of nonpositive curvature, Birkhäuser, Basel-Boston, 1985.

[BG] K. Burns and M. Gerber, Real analytic Bernoulli geodesic flows on $S^{2}$, Indiana Univ., preprint.

[BO] R. Bishop and B. O’Neill, Manifolds of negative curvature, Trans. Amer. Math. Soc. 145 (1969), $1-49$.

[G] M. Gromov, Hyperbolic manifolds according to Thurston and Jørgensen, Sém. Bourbaki 546 (1979), Springer Lecture Notes in Math. 842, 40-53.

[S] V. Schroeder, Existence of immersed tori in manifolds of nonpositive curvature, J. Reine Angew. Math., 390 (1988), 32-46.

[T] W. Thurston, The geometry and topology of 3-manifolds, Lecture Notes, Princeton Univ., $1977 / 78$.

Department of Mathematics, Mathematisches Institut, Hebelstr. 29, 7800 Freiburg, West Germany 